\title{
FREE ELECTRONS OUTSIDE HII REGIONS
}

\author{
M. GUÉLIN \\ Observatoire de Paris, Meudon, France
}

\begin{abstract}
This paper reviews the recent observational results on the electron distribution outside the HII regions. The mean electron density in the solar neighbourhood is of the order of $0.03 \mathrm{~cm}^{-3}$, and does not seem to vary very much between the HI clouds and the intercloud medium, or between the arms and the interarm region. The $z$ thickness of the electron layer in the solar neighbourhood is found to be larger than or of the order of $0.8 \mathrm{kpc}$. No reliable figure of the electron distribution can be deduced for the moment in the inner parts of the Galaxy.
\end{abstract}

\section{Introduction}

In directions of the sky where no discrete $\mathrm{H}$ II region can be recognized from the radio continuum maps, a number of radio or optical observations show the presence of ionized interstellar matter.

The aim of this review is to try to determine which part of this matter is concentrated in the small $\mathrm{H}$ II regions which may be crossed by the line of sight, and which part refers to what we shall call the 'general' interstellar gas, that is to say the hot or cold gas only partly ionized, outside the Strömgren spheres of the hot stars. As will be seen below, this distinction is not always clear, in particular because the characteristics of possible $\mathrm{H}$ II regions surrounding the B stars (for instance the electron density) are badly known. Hence, while trying to avoid the large emission nebulae, we shall deal with data relative to these weak $\mathrm{H}$ II regions as well as to the general medium.

We shall review the recent important results inferred from:

(i) the pulsar dispersion measure and distance measurements; (ii) the interstellar absorption lines; (iii) the radio recombination lines and the free-free absorption, and (iv) the diffuse $\mathrm{H} \alpha$ and $[\mathrm{N}$ II] emissions.

The pulsar dispersion measurements give clear-cut information on the average electron density in interstellar space. The other observations yield quantities depending both on electron density and on temperature. These two parameters cannot be properly separated, and apart from the interstellar absorption lines and some recombination lines which clearly originate in cold gas, these observations are for the moment unreliable for giving either the density or the temperature of the emitting electrons.

\section{Pulsar Dispersion Measure and Distance}

A straightforward determination of the mean electron density $n_{\mathrm{e}}$ in interstellar space can be made in the direction of the pulsars for which we know the dispersion measure and the distance. The dispersion measure, $D M=\int n_{\mathrm{e}} \mathrm{d} l$, which represents the electron column density in the line of sight, can be generally derived with good accuracy (see, 
e.g., McCulloch et al., 1973). This has been done now for about a hundred pulsars. The dependence of their dispersion measure on galactic latitude shows clearly that, in the mean, most of the dispersion occurs in the interstellar medium and not in the immediate vicinity of the pulsars.

The determination of distance is much more difficult. It results from the analysis of the 21-cm line absorption of the pulsar signal, or, as for the Crab nebula pulsar, from the association with a supernova remnant of known distance. As most of the pulsars are very faint at $21-\mathrm{cm}$ wavelength, the absorption method can be applied only to a few sources. As an example, Figure 1 shows the absorption spectrum of
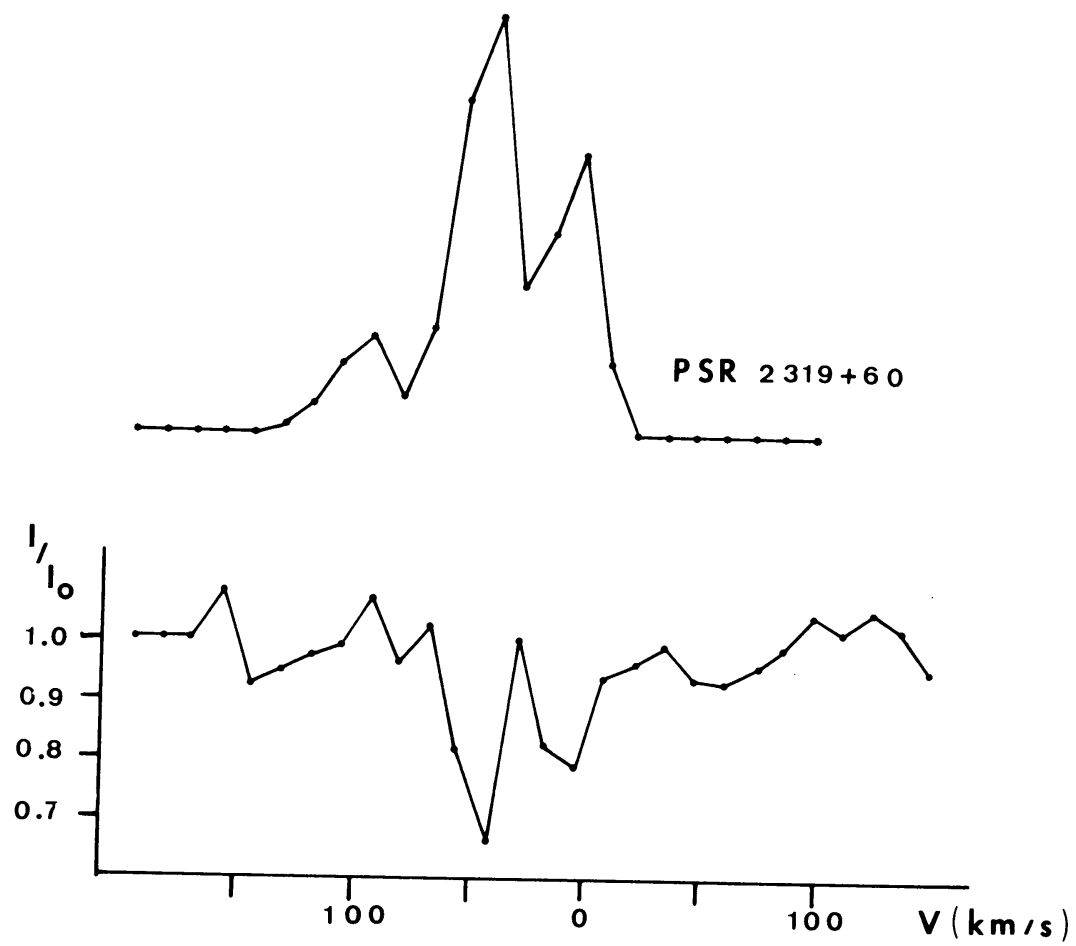

Fig. 1. Emission (upper) and absorption (lower) spectra in the direction of pulsar PSR $2319+60$. These spectra were obtained with the Nançay radio telescope using a $12.7 \mathrm{~km} \mathrm{~s}^{-1}$ velocity resolution. The absorption features near $0 \mathrm{~km} \mathrm{~s}^{-1}$ and $-40 \mathrm{~km} \mathrm{~s}^{-1}$ correspond to absorption in the local and Perseus arms respectively.

PSR $2319+60$ which was obtained with the Nançay radio telescope after $20 \mathrm{~h}$ of observation. Absorption is clearly present at $0 \mathrm{~km} \mathrm{~s}^{-1}$ and $-40 \mathrm{~km} \mathrm{~s}^{-1}$, the velocities of the local and Perseus arms. This indicates that PSR $2319+60$ is located farther than $3 \mathrm{kpc}$ in the Schmidt galactic rotation model.

Table I lists the pulsars for which distances (or limits to distance) are known independently of the dispersion measure. The distances of PSR $0531+21,0611+22$ and 0833-45 are taken equal to the distances of the Crab nebula (Trimble, 1968), IC 443 
TABLE I

Pulsars with known distances

\begin{tabular}{lrrlll}
\hline Name & $l$ & $b$ & $\begin{array}{l}D M \\
\left(\mathrm{~cm}^{-3} \mathrm{pc}\right)\end{array}$ & $\begin{array}{l}\text { Distance } \\
(\mathrm{pc})\end{array}$ & $\begin{array}{l}\left\langle n_{\mathrm{e}}\right\rangle \\
\left(\mathrm{cm}^{-3}\right)\end{array}$ \\
\hline PSR 0329+54 & 145 & -1 & 26.8 & $1000<D<2000$ & $0.015<n_{\mathrm{e}}<0.03$ \\
PSR 0525+21 & 184 & -7 & 51.0 & 2000 & 0.025 \\
PSR 0531+21 & 185 & -6 & 56.8 & 2000 & 0.03 \\
PSR 0611+22 & 189 & 2 & 99 & 2000 & 0.05 \\
PSR 0740-28 & 244 & -2 & 73.8 & $1500<D<2500$ & $0.03<n_{\mathrm{e}}<0.06$ \\
PSR 0833-45 & 264 & -3 & 69.2 & 500 & $0.14($ Gum Neb- \\
PSR 1749-49 & 1 & -1 & 50.9 & $D<1000$ & $0.05<n_{\mathrm{e}} \quad$ ula) \\
PSR 1818-04 & 26 & 5 & 84.4 & $D<1500$ & $0.06<n_{\mathrm{e}}$ \\
PSR 1929+10 & 47 & -4 & 3.2 & $D<1000$ & $0.003<n_{\mathrm{e}}$ \\
PSR 1933+16 & 52 & -2 & 158.5 & $6000<D$ & $n_{\mathrm{e}}<0.025$ \\
PSR 2016+28 & 68 & -4 & 14.2 & $1000<D$ & $n_{\mathrm{e}}<0.015$ \\
PSR 2020+28 & 69 & -5 & 44 & $1000<D$ & $n_{\mathrm{e}}<0.04$ \\
PSR 2021+51 & 88 & 8 & 22.6 & $D<1000$ & $0.02<n_{\mathrm{e}}$ \\
PSR 2319+60 & 112 & 1 & 96 & $3000<D$ & $n_{\mathrm{e}}<0.03$ \\
\hline
\end{tabular}

(Minkowsky, 1959) and Vela X (Ilovaisky and Lequeux, 1972) respectively. The other pulsar distances result from $21-\mathrm{cm}$ line absorption profiles observed at Nançay (Guélin, 1973; Guélin and Gomez-Gonzalez, 1974).

The range of variation of the line-of-sight-averaged electron density, $\left\langle n_{\mathrm{e}}\right\rangle$, must be derived with care from Table I, as in most of the cases only limits to $\left\langle n_{\mathrm{e}}\right\rangle$ are reported. These limits are very loose in the case of nearby pulsars such as PSR 1929 +10 , because the relative errors in their distances are large. Nevertheless, the mean electron density appears to vary at least between 0.015 and $0.14 \mathrm{~cm}^{-3}$. This scatter is reduced if we only consider the distant sources. Rough lower limits to $\left\langle n_{\mathrm{e}}\right\rangle$ towards PSR $1933+16$ and $2319+60$ can be set by assuming that these pulsars do not lie farther than $15 \mathrm{kpc}$ from the galactic centre. Using the upper limits of Table I, we then derive $0.01<n_{\mathrm{e}}<0.025 \mathrm{~cm}^{-3}$ for PSR $1933+16$ and $0.015<n_{\mathrm{e}}<0.03 \mathrm{~cm}^{-3}$ for PSR $2319+60$. These values, as well as those of Table I in the direction of the other distant pulsars, agree within a factor of two with the mean value of $0.03 \mathrm{~cm}^{-3}$ which may be derived from Table $I$. As these distant sources are scattered over a large range of galactic longitude, this suggests a roughly uniform large-scale electron density in the solar neighbourhood. The limits of 0.015 and $0.14 \mathrm{~cm}^{-3}$ show, on the other hand, relatively important local variations.

To derive the average electron density outside the $\mathrm{H}$ il regions, one has first to check whether the line of sight to a pulsar cuts across the Strömgren sphere of known very hot stars or clusters of hot stars. They are few in the solar vicinity and this check can be made relatively easily (Prentice and ter Haar, 1969). For instance, the line of sight to PSR $0833-45$ very probably crosses some large $\mathrm{H}$ II regions in the Gum nebula. Thus, the average value of $n_{\mathrm{e}}$ is unusually high in this direction and has not to be taken into account in the estimate of $n_{\mathrm{e}}$ for the general medium.

This discussion becomes much more difficult when we go further away from the 
Sun or when we consider the possible contribution of cooler stars like B stars (Grewing and Walmsley, 1971). Simple considerations, however, suggest that the contribution of the 'classical' $\mathrm{H}$ II regions is relatively small in the mean.

Let us consider the pulsars of known dispersion measure, and among them, those with $D M \cos |b|<30 \mathrm{~cm}^{-3} \mathrm{pc}$ ( $b$ is the galactic latitude). Because of the mean value of $\left\langle n_{\mathrm{e}}\right\rangle$ derived above, most of these pulsars lie in a region one kiloparsec in radius centred on the Sun. In this region, we know the distribution of the $O$ and $B$ type stars and we can recognize those pulsars for which the line of sight may cross the Strömgren sphere of one of these stars and those pulsars for which this is unlikely. This separation depends on the size of the Strömgren spheres and thus on the density inside the $\mathrm{H}_{\mathrm{II}}$ regions. Assuming that the latter is not smaller than $0.5 \mathrm{~cm}^{-3}$, one remains with about twenty-five 'non-contaminated' cases.

If, on the average, the contribution of $\mathrm{H}$ II regions to the pulsar dispersion measures were important, $\left\langle n_{\mathrm{e}}\right\rangle$ would be substantially smaller along the 'clean' lines of sight than along the 'contaminated' ones. Thus, the distances and heights above the galactic plane, $z$, derived from the dispersion measures by using $\left\langle n_{\mathrm{e}}\right\rangle=0.03 \mathrm{~cm}^{-3}$ would be strongly underestimated for the 'clean' cases. In fact, the $z$ distribution of the 'clean' pulsars is very similar to that of the other pulsars and the mean scale height $\langle|z|\rangle$ is very close to $200 \mathrm{pc}$ for the 'clean' nearby pulsars as well as for the remaining nearby ones or for the distant ones.

The same type of considerations shows that there is probably no large difference between $\left\langle n_{\mathrm{e}}\right\rangle$ in the arms and in the interarm region comprised between longitudes $30^{\circ}$ and $50^{\circ}$.

We will thus tentatively conclude that in the solar neighbourhood, the mean electron density in the general interstellar medium is about $0.03 \mathrm{~cm}^{-3}$ and is, on a scale of a few kiloparsecs, constant within a factor two. The resulting $z$ distribution of all the pulsars of known dispersion measure (Figure 2) shows moreover that the electron layer is thicker than $0.8 \mathrm{kpc}$. This is in good agreement with the determinations resulting from free-free absorption or Faraday rotation measurements (Bridle and Venugopal, 1969; Falgarone and Lequeux, 1973).

A more detailed electron density distribution cannot be derived in this way as pulsar distance estimates are inaccurate (typical uncertainties are of the order of $1 \mathrm{kpc})$.

\section{Interstellar Absorption Lines}

Observation of interstellar absorption lines in front of hot stars offers an opportunity to study the electron density on a smaller scale. Measurements of the relative abundance of different ions of the same atom lead to the electron density, under the usual assumption of ionization equilibrium, provided that the recombination coefficient and the ionization rate are known. If the absorption lines originate in concentrations similar to those detected in the 21-cm line absorption surveys (Radhakrishnan et al., 1972), the recombination coefficient and the ionization rate can be properly estimated, probably within a factor of 3 . The electron densities derived in this way are typically 


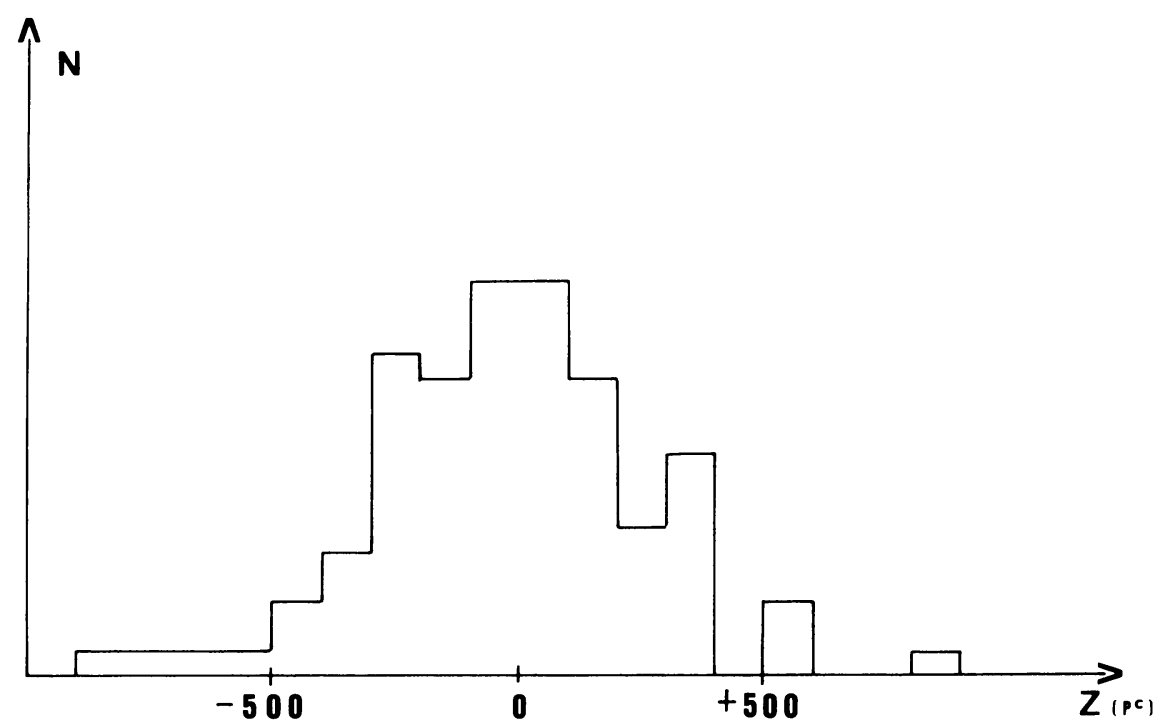

Fig. 2. Distribution of pulsars in galactic $z$-distance. The distances are derived from the dispersion measures by using $\left\langle n_{\mathrm{e}}\right\rangle=0.03 \mathrm{~cm}^{-3}$.

a few times $10^{-2} \mathrm{~cm}^{-3}$ (White, 1973; Jenkins, 1974), thus close to the average value in the interstellar medium derived from pulsar measurements. Since the cold $\mathrm{HI}$ concentrations fill only a small fraction of the line of sight, this means that $n_{\mathrm{e}}$ does not vary very much between the clouds and the intercloud medium.

\section{Hydrogen and Carbon Radio Recombination Lines in Cold Clouds}

Estimates of the electron density and temperature can also be derived from the radio recombination lines of hydrogen, carbon and heavier elements in some cool gas concentrations. The comparison of the width of the hydrogen lines with that of the carbon lines, assuming that the two emissions originate in the same region, allows us in principle to separate thermal broadening from turbulence. Chaisson and Lada (1974) have recently done this for four clouds and find typically $400 \pm{ }_{400}^{1000} \mathrm{~K}$. The uncertainties are too large to show anything else than that these lines do not arise in $\mathrm{H} \mathrm{II}$ regions. On the other hand, the ratio of intensities of the carbon and hydrogen lines may lead to the relative abundance of the electrons, $n_{\mathrm{e}} / n_{\mathrm{H}}$, provided that carbon ions and hydrogen ions share the same volume in the cool region. This assumption, of course, is very questionable. Assuming moreover that the abundances of carbon and heavier elements are equal to or smaller than the cosmic abundances, Chaisson and Lada find $n_{\mathrm{e}} / n_{\mathrm{H}} \leqslant 10^{-3}$.

\section{Diffuse Radio Recombination Line Emission from the Galactic Plane}

In opposition to these narrow hydrogen and carbon lines which are observed only 
in front of some strong $\mathrm{H}$ il regions, broad hydrogen recombination lines have been observed from regions of the galactic plane free of discrete radiosources.

Figure 3 summarizes the observations (Gordon and Cato, 1972; Gordon et al., 1972; Matthews et al., 1973; Jackson and Kerr, 1974). The observed directions are plotted on a neutral hydrogen longitude-velocity map (Burton, 1971). The heavy lines indicate the velocity interval explored and the dashed portions the velocity extent of the lines when detected. Three points have to be made. First, the width of the lines is large compared with what may be expected from the emission of a single region; this suggests that the radiation originates in different regions widely spread over the

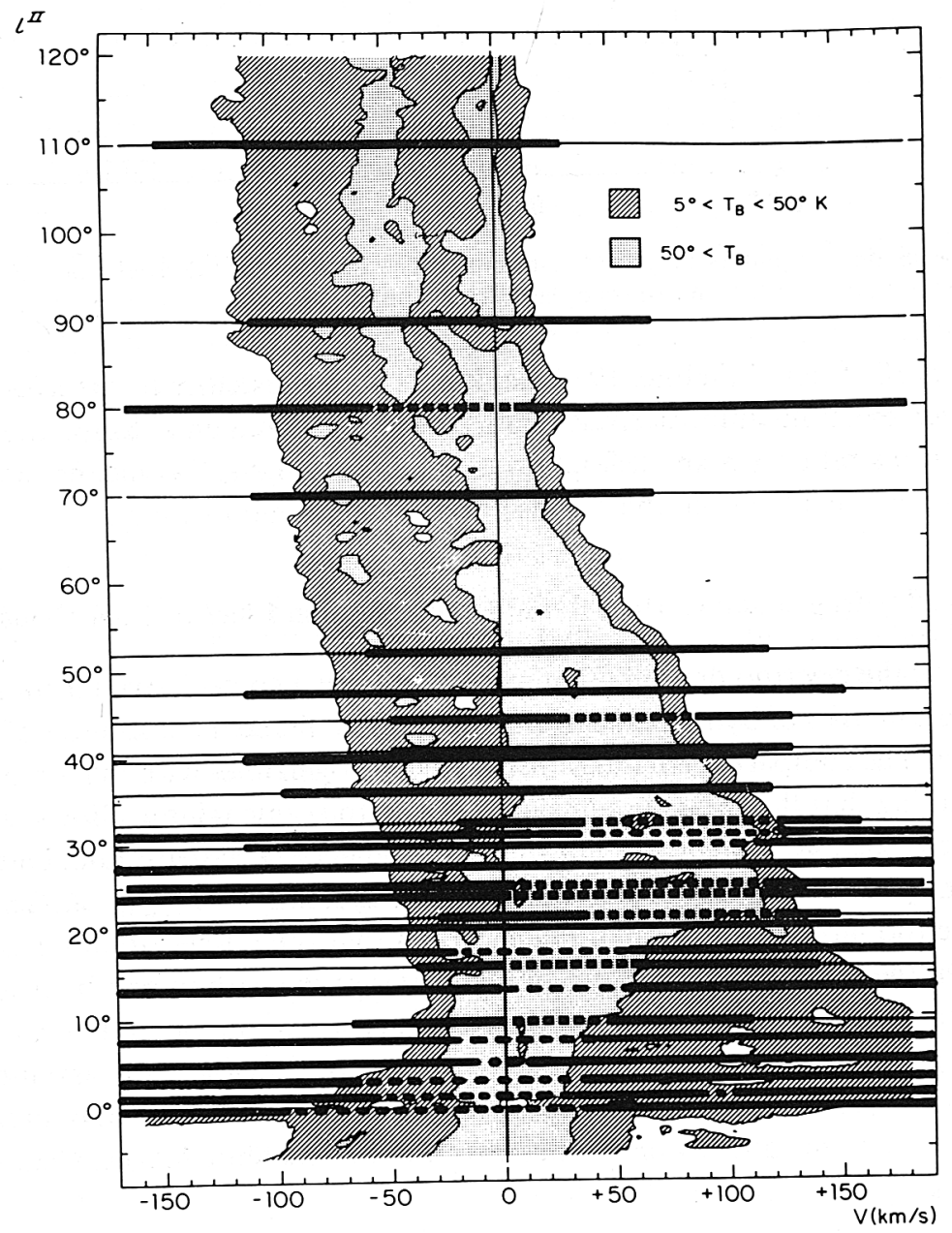

Fig. 3. Diffuse radio recombination emission from the galactic plane. The observed directions (Gordon and Cato, 1972; Matthews et al., 1973; Jackson and Kerr, 1974) are plotted on a neutral hydrogen longitudevelocity map (Burton, 1971). The heavy lines indicate the velocity interval explored and the dashed portions the velocity extent of the lines. 
line of sight. Second, the diffuse line emission is detected in nearly all the observed directions with longitude smaller than $30^{\circ}$ and nearly disappears for $l \geqslant 35^{\circ}$. Third, the latitude extent of the line (at least near $l=33^{\circ}$ ) is narrower than $1^{\circ}$. The diffuse recombination emission appears thus to be mainly concentrated in the zone of strong radio continuum emission from the galactic plane $\left(l<35^{\circ},|b|<1^{\circ}\right)$. In the same way, the broad band line emission observed at $l=80^{\circ}$ in Cygnus also coincides with a zone of strong continuum. More generally, Matthews et al. (1973) and Jackson and Kerr (1974) have found that, in fact, the line strength of the radio recombination profiles is correlated with the temperature of the continuum at $6-\mathrm{cm}$ wavelength, thus probably with the thermal radiation.

The question now arises whether these lines are predominantly emitted by unresolved $\mathrm{H}$ II regions, or by the general interstellar medium, and what are the temperature and density associated with the ionized gas.

The line strength of the radio recombination emission yields directly the integral:

$$
\int n_{\mathrm{e}}^{2} T_{\mathrm{e}}^{-1.5} \mathrm{~d} l
$$

in the case of local thermodynamic equilibrium and of low optical depth (Gordon and Cato, 1972). In order to separate $n_{\mathrm{e}}$ and $T_{\mathrm{e}}$, one has to compare this integral with a different function of $n_{\mathrm{e}}$ and $T_{\mathrm{e}}$, measured along the same line of sight and, as far as possible, with the same angular resolution. This is usually done in two ways: (i) with the help of free-free thermal emission measurements, or (ii) with the help of free-free absorption measurements.

In the case of low optical depth and LTE, the ratio $\varrho$ of the line strength integral to the thermal continuum temperature may be expressed as:

$$
\varrho \sim \frac{\int n_{\mathrm{e}}^{2} T_{\mathrm{e}}^{-1.5} \mathrm{~d} l}{\int n_{\mathrm{e}}^{2} T_{\mathrm{e}}^{-0.35} \mathrm{~d} l},
$$

or, if the emitting gas is isothermal,

$$
\varrho \sim T_{\mathrm{e}}^{-1.15}
$$

Using this ratio, Matthews et al. (1973) and Jackson and Kerr (1974) find temperatures ranging from 4000 to $8000 \mathrm{~K}$, which leads to emission measures $E M=\int n_{\mathrm{e}}^{2} \mathrm{~d} l$ of a few times $10^{3} \mathrm{~cm}^{-6} \mathrm{pc}$ in a $10 \mathrm{kpc}$ path.

These estimates assume however: (i) that the lines are formed in LTE; (ii) that the thermal continuum is accurately evaluated; and (iii) that the emitting region is isothermal. These assumptions, particularly the last one, make the derived $T_{\mathrm{e}}$ and $E M$ very tentative.

On the other hand, the comparison of the radio recombination line power to the free-free absorption in the same direction may also allow one to evaluate the temperature if the emitting gas is isothermal. This has been tried by Cesarsky and Cesarsky (1973a) who have observed the H92 $\alpha$ emission in the direction of the super- 
nova remnant 3C 391 and compared their results to the free-free absorption measurements of this source (Dulk and Slee, 1972). At the frequency of their observations $(8.3 \mathrm{GHz})$, the ratio $\varrho^{\prime}$ of the recombination line power to the free-free optical depth becomes strongly dependent on the temperature. Cesarsky and Cesarsky obtain a very low value of $\varrho^{\prime}$, which means that the radio recombination line originates in a low temperature medium $\left(20_{-20}^{+300} \mathrm{~K}\right)$, that is to say in the cold clouds. This conclusion is somewhat supported by the detection, in the same direction, of a line which is probably due to recombination of carbon (Cesarsky and Cesarsky, 1973b).

This temperature estimate, which contradicts the previous ones, seems however not very reliable since: (i) the temperature could be as high as $10^{4} \mathrm{~K}$ if the actual error in $\varrho^{\prime}$ is only two times its quoted value; and (ii) the correlation of the freefree emission with the recombination line emission on the one hand, as well as the high resolution observations on the other hand (Altenhoff et al., 1973; Jackson and Kerr, 1974), suggest that part of the 'diffuse' recombination line emission arises in a hot medium.

Altenhoff et al. (1973) have mapped, at $11-\mathrm{cm}$ wavelength and with a $4.5^{\prime}$ resolution, one of the typical regions where radio recombination lines of large velocity extent have been reported by Gottesman and Gordon (1970) from 18' resolution observations. Within $36^{\prime}$ of the Gottesman and Gordon position $\left(l=24^{\circ}\right)$, they have found ten discrete radiosources. At least three of these sources show radio recombination line emission with characteristics typical of $\mathrm{H}$ II regions (half-power line-width of $25 \mathrm{~km} \mathrm{~s}^{-1}$ and line-to-continuum ratio of about $3 \%$ ); whereas between the sources a weak and broad line emission is probably still present (half-power line-width of $80 \mathrm{~km} \mathrm{~s}^{-1}$ ). Thus, at least part of the emission reported originally by Gottesman and Gordon at this position arises in normal $\mathrm{H}$ II regions. But at the same time, it remains possible that emission from $\mathrm{H}_{\mathrm{I}}$ regions may be responsible at least partly for the weak and broad background line radiation seen between the patches of continuum and recombination line emission.

\section{Weak $\mathrm{H} \alpha$ and [N II] Emission from the Galactic Plane}

Weak diffuse $\mathrm{H} \alpha$ and [ $\mathrm{N}$ II] emission has been detected towards many directions between $\pm 30^{\circ}$ of the galactic plane (Reynolds et al., 1973; Monnet, 1974). It has been investigated in detail by Reynolds, Scherb and Roesler between galactic longitudes $0^{\circ}$ and $120^{\circ}$.

A very good correlation exists in velocity between this emission and the $21-\mathrm{cm}$ line emission from the H I gas nearer than $3 \mathrm{kpc}$ (see Reynolds et al., 1973). This shows that the emitting regions are spread in the neutral hydrogen arms. A good correlation exists also between the distribution in the sky of the brightest of these $\mathrm{H} \alpha$ features and the distribution of the emission nebulae (Lynds, 1965). This is particularly clear for the Cygnus $\left(l=80^{\circ}\right)$ and the Ophiucus $\left(l=10^{\circ}\right)$ regions where the bulk of the $\mathrm{H} \alpha$ emission very probably is related to the $\mathrm{H}$ II regions.

$\mathrm{H} \alpha /[\mathrm{N} \mathrm{II}]$ intensity ratios have been observed in the direction of Orion. They range 
from 2 to 5 and are thus similar to those found in typical $\mathrm{H}$ il regions. In the same way, the width of the lines in the anticenter direction implies temperatures of the order of 6000 to $8000 \mathrm{~K}$, provided that no turbulent motions larger than $7 \mathrm{~km} \mathrm{~s}^{-1}$ occur. Assuming a temperature of $6000 \mathrm{~K}$ and correcting the observed intensities for visual extinction, Reynolds et al.(1973) find emission measures in a $2 \mathrm{kpc}$ path ranging from $10^{2}$ to a few times $10^{3} \mathrm{~cm}^{-6} \mathrm{pc}$.

A similar $\mathrm{H} \alpha$ emission has been detected in external galaxies (Comte, 1973). Figure 4a represents an $\mathrm{H} \alpha$ filter photograph of the southern spiral arm of M33, compared with a Pérot-Fabry $\mathrm{H} \alpha$ interferogram of the same region (Figure $4 \mathrm{~b}$ ). The stellar con-

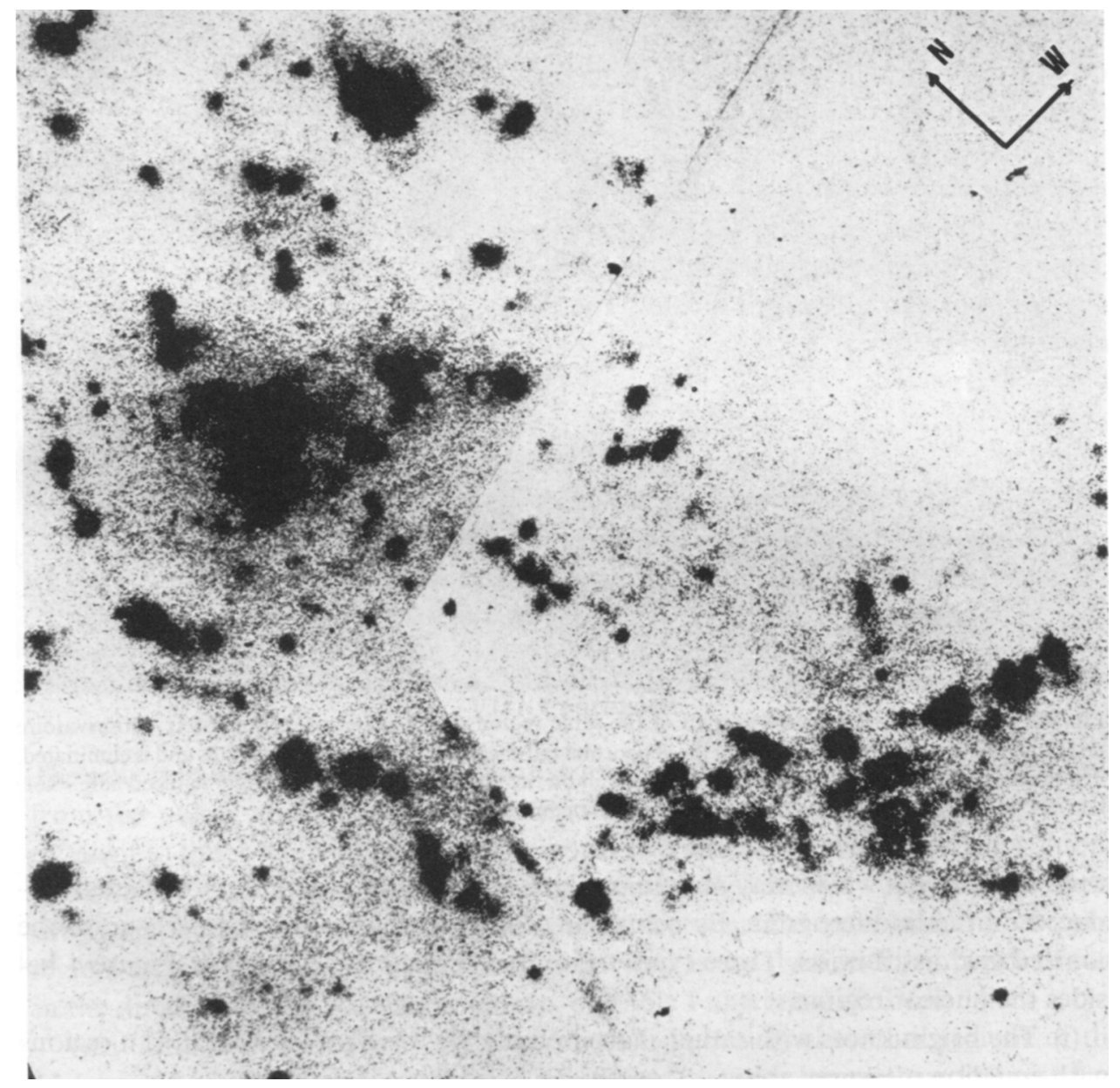

Fig. 4a. H $\alpha$ filter photograph of the southern arm of M33 (Boulesteix, Observatoire de Haute Provence). The field of the photograph is $15^{\prime}$. The large emission region in the left part of the photograph is the centre of the galaxy. Note the diffuse or filamentary structure which appears in the spiral arm between the bright $\mathrm{H}$ II regions. This diffuse emission looks very similar to the diffuse $\mathrm{H} \alpha$ emission detected in our Galaxy

(Reynolds et al., 1973). 


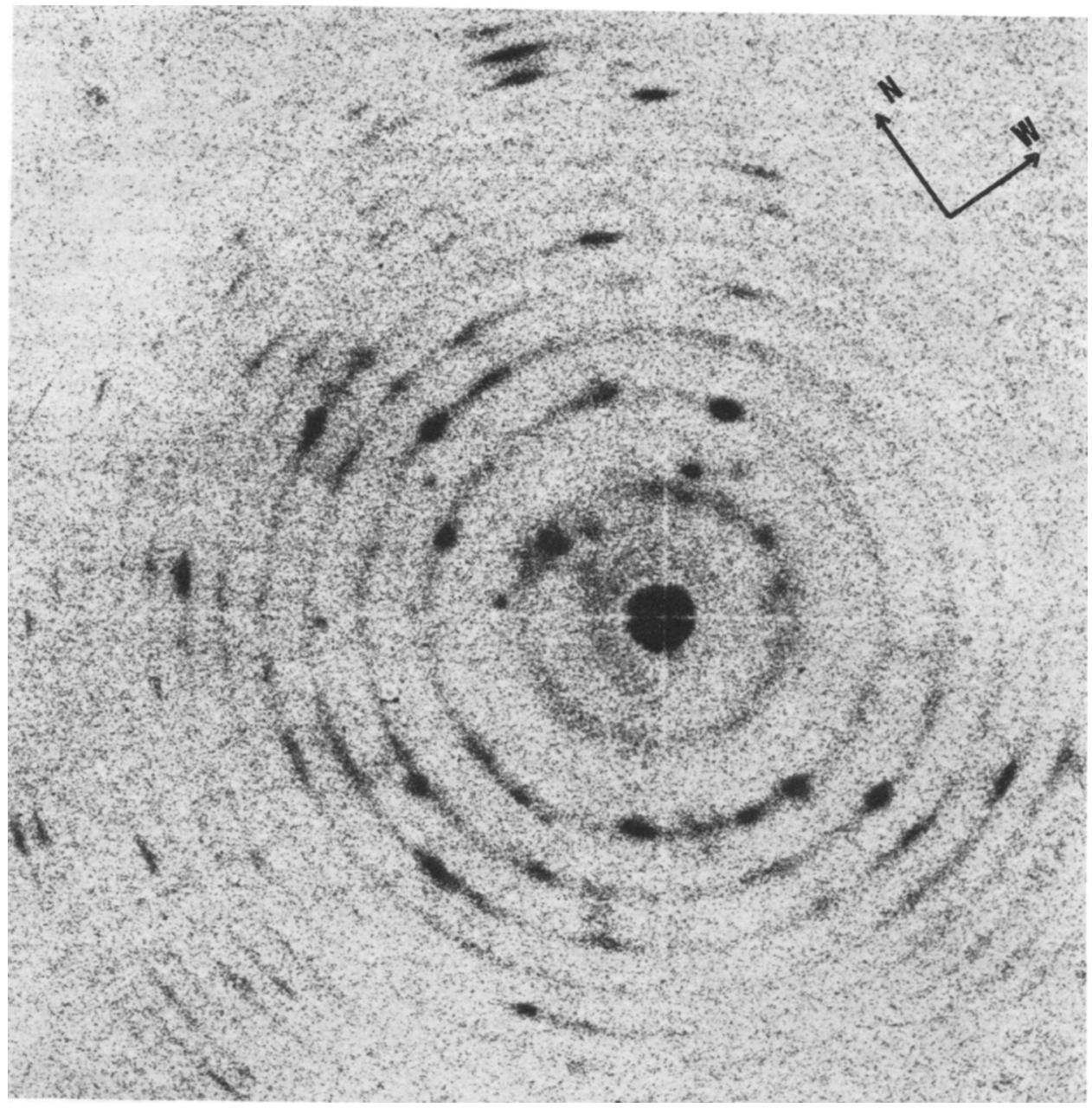

Fig. 4b. H $\alpha$ Pérot-Fabry interferogram of the same region to the same scale (Boulesteix, Observatoire de Haute Provence). $\mathrm{H} \alpha$ emission from the geocorona falls between two Pérot-Fabry rings and is eliminated. The stellar continuum radiation which may remain on the filter photograph is strongly reduced here. Note that the diffuse filaments are still present.

tinuum radiation which may remain on the $6 \AA$ passband photograph is strongly reduced on the interferogram. By comparing the two pictures, one can recognize the ionized gas distribution. Three types of ionized regions are present in Figure 4 besides the nuclear region:

(i) The bright knots with rather sharp boundaries which are 'classical' $\mathrm{H}$ II regions with emission measures about $10^{4}$ to $10^{5} \mathrm{~cm}^{-6} \mathrm{pc}$;

(ii) between these knots, a relatively bright filamentary structure which appears only in the arms, with $\mathrm{H} \alpha /[\mathrm{N} \mathrm{II}]$ and $\mathrm{H} \alpha /[\mathrm{S} \mathrm{II}]$ ratios similiar to those in the knots and with emission measures typically about $10^{2} \mathrm{~cm}^{-6} \mathrm{pc}$ for a temperature of $6000 \mathrm{~K}$ (Comte, 1973); 
(iii) a general background much weaker than the filamentary structure and with $\mathrm{H} \alpha$ to $[\mathrm{N} \mathrm{II}]$ and $[\mathrm{S} \mathrm{II}]$ ratios much smaller than in the knots.

The diffuse filamentary structure of M33 appears very like the weak galactic $\mathrm{H} \alpha$ structure studied by Reynolds, Scherb and Roesler: (i) both have comparable $\mathrm{H} \alpha$ intensities, if one allows for the difference in path length; (ii) both appear in spiral arms and in the vicinity of $\mathrm{H}$ II regions, and (iii) they present comparable $\mathrm{H} \alpha /[\mathrm{N}$ II $]$ ratios. Because of their similarity with the classical $\mathrm{H}$ II regions and of their location in the arms, Comte has identified the filaments of M33 with extended $\mathrm{H}$ il regions, excited by ultraviolet radiation escaping from bright nearby $\mathrm{H}$ il regions. An alternative explanation may be numerous small $\mathrm{H}$ II regions surrounding relatively cool stars. In both cases the filaments are hot, and the emission measure across one filament amounts to a few times $10^{2} \mathrm{~cm}^{-6} \mathrm{pc}$ in the mean. On the other hand, scattering of $\mathrm{H} \alpha$ and [N II] light from the bright $\mathrm{H}_{\text {II }}$ regions by the interstellar grains may also contribute substantially to this emission. The same explanations may apply for the diffuse $\mathrm{H} \alpha$ emission in our Galaxy.

If actually this diffuse $\mathrm{H} \alpha$ emission arises in hot gas (its probable association with [N II] emission argues for temperatures larger than $3000 \mathrm{~K}$ ), the emission measure along a $2 \mathrm{kpc}$ path towards Sagittarius, in directions free of any strong $\mathrm{H}$ II region, is of the order of $10^{3} \mathrm{~cm}^{-6} \mathrm{pc}$ (Reynolds et al., 1973). From this, one can predict a radio recombination integrated brightness temperature of a few $\mathrm{K} \mathrm{kHz}$ at $18-\mathrm{cm}$ wavelength. In fact, the values observed in the same directions (Gottesman and Gordon, 1970) appear very comparable to this figure if one takes into account that the radio intensities refer to a path length of about $10 \mathrm{kpc}$. This discussion, however, remains tentative as important extinction corrections have to be applied to the optical intensities in the Sagittarius region, and as the radio intensities, which may be predicted from the observed $\mathrm{H} \alpha$ intensities in directions of small visual extinction, are below the sensitivity of the present surveys.

\section{Conclusion}

The only reliable data on the electron distribution outside the $\mathrm{H}$ il regions result thus from the pulsar and interstellar absorption line measurements. They refer for the moment to the solar neighbourhood and only to a small number of directions. Along these directions, it appears that the electron density does not vary very much between the $\mathrm{H}$ I clouds and the intercloud medium or between the arms and the interarm region. Its value, averaged over a few kiloparsecs, is constant within a factor of two in the direction of the six distant pulsars of Table I and equals $0.03 \mathrm{~cm}^{-3}$.

Very little is known about the electron distribution in the other regions of the Galaxy. About twenty pulsars have a dispersion measure larger than $150 \mathrm{~cm}^{-3} \mathrm{pc}$ and are thus very probably more distant than 4 or $5 \mathrm{kpc}$ from the Sun. Their scale height above the galactic plane, $\langle|z|\rangle$, derived from their dispersion measures by assuming a constant electron density, is very close to that of the nearby pulsars. This suggests that even at distances of the order of $4 \mathrm{kpc}$, the mean electron density re- 
mains of the order of $0.03 \mathrm{~cm}^{-3}$. The small number of high dispersion pulsars prevents however extending this argument to more distant regions. No one of the pulsars of Table $I$ is located in the inner part of the Galaxy so that no direct determination of the electron density can be made in this region. Diffuse radio recombination lines are observed in the central region of the Galaxy but, as has been seen, they come in an unknown proportion from normal $\mathrm{H}$ il regions, and are thus unreliable for giving either the temperature or the density in the general medium.

\section{References}

Altenhoff, W., Churchwell, E. B., Mebold, U., and Walmsley, M.: 1973, private communication. Bridle, A. H. and Venugopal, V. R.: 1969, Nature 224, 545.

Burton, W. B.: 1971, Astron. Astrophy. 10, 76.

Cesarsky, D. A. and Cesarsky, C. J.: 1973a, Astrophys. J. 184, 83.

Cesarsky, D. A. and Cesarsky, C. J.: 1973b, Astrophys. J. Letters 183, L143.

Chaisson, E. J. and Lada, C. J.: 1974, Astrophys. J. 189, 227.

Comte, G.: 1973, Thèse de 3eme cycle Université de Provence.

Dulk, G. A. and Slee, O. B. : 1972, Australian J. Phys. 25, 429.

Falgarone, E. and Lequeux, J.: 1973, Astron. Astrophys. 25, 253.

Gordon, M. A. and Cato, T.: 1972, Astrophys. J. 176, 587.

Gordon, M. A., Brown, R. L., and Gottesman, S. T.: 1972, Astrophys. J. 178, 119.

Gottesman, S. T. and Gordon, M. A.: 1970, Astrophys. J. Letters 162, L93.

Grewing, M. and Walmsley, M.: 1971, Astron. Astrophys. 11, 65.

Guélin, M.: 1973, Proc. Inst. Elec. Electron. Engrs. 61, 1298.

Guélin, M. and Gomez Gonzalez, J.: 1974, Astron. Astrophys. 32, 441.

Ilovaisky, S. A. and Lequeux, J.: 1972, Astron. Astrophys. 20, 347.

Jackson, P. D. and Kerr, F. J.: 1974, Astrophys. J., in press.

Jenkins, E. B. : 1974, this volume, p. 65.

Lynds, B. T.: 1965, Astrophys. J. Suppl. 12, 163.

McCulloch, P. M., Komesaroff, M. M., Ables, J. G., Hamilton, P. A., and Rankin, J. M. : 1973, Astrophys. J. Letters 14, 169.

Matthews, H. E., Pedlar, A., and Davies, R. D.: 1973, Monthly Notices Roy. Astron. Sac. 165, 149.

Minkowski, R.: 1959, in R. N. Bracewell (ed.), 'Paris Symposium on Radio Astronomy', IAU Symp. 9, 315.

Monnet, G.: 1974, this volume, p. 249.

Prentice, A. J. R. and ter Haar, D.: 1969, Monthly Notices Roy. Astron. Soc. 146, 423.

Radhakrishnan, V., Murray, J. D., Lockhart, P., and Whittle, R. P. J.: 1972, Astrophys. J. Suppl. 24, 15.

Reynolds, R. J., Scherb, F., and Roester, F. L.: 1973, Astrophys. J. 185, 869.

Trimble, V. L.: 1968, Astron. J. 73, 535.

White, R. E.: 1973, Astrophys. J. 183, 81.

M. Guélin

Observatoire de Paris,

Section d'Astrophysique de Meudon,

92190 Meudon, France

\section{DISCUSSION}

Gordon: It is important not to compare available $\left\langle n_{\mathrm{e}}\right\rangle$ derived from pulsars and from radio recombination lines. While dispersions are seen in pulsars from a range of longitudes, radio recombination line emission is seen only from directions in which the line of sight lies inside the solar radius. No recombination lines have been seen in the direction of pulsars. The data imply that the dispersion measures and the lines arise from regions of vèry different characteristics. 
Guélin: I agree. The dispersion measures depend on $n_{\mathrm{e}}$ whereas the radio recombination lines depend on $n_{\mathrm{e}}^{2}$.

Zuckerman: You mentioned the observation of carbon and hydrogen recombination lines by Chaisson and his co-workers and indicated that these observations have been used to deduce the fraction of hydrogen that is ionized in HI regions. As I will discuss in my talk that follows, I do not believe that these recombination lines can be used in this way, because the hydrogen and carbon lines both come from very dense regions and, thus, may not be coextensively ionized. Furthermore, as we have seen from the Copernicus results, one may not assume that a unique 'cosmic' $\mathrm{C} / \mathrm{H}$ ratio generally obtains in interstellar clouds. Finally, the carbon lines originate in very dense clouds near $\mathrm{H}$ II regions, and these special clouds are hardly representative of the type of regions that you were otherwise discussing.

Terzian: I hope that we do not go away from here and try to use $n_{\mathrm{e}}=0.03 \mathrm{~cm}^{-3}$ to derive distances for all the pulsars. In an analysis of pulsar dispersion measures which we have performed we find a range of $n_{\mathrm{e}}$ from 0.01 to $0.1 \mathrm{~cm}^{-3}$, with a mean of $\sim 0.04 \mathrm{~cm}^{-3}$.

Guélin: Many people and you too have made some estimates of distances of pulsars by using the dispersion measure. These were done before the $21-\mathrm{cm}$ line absorption measurements. Now every time we get a measure by the $21-\mathrm{cm}$ line, the dispersion measure distance appears to be wrong.

Greenberg: How do you take into account the possibility that the $\mathrm{O}$ and B stars do not sit in a cloud but rather in an intercloud region? The Strömgren sphere then extends far beyond its 'normal' distance from the star, i.e., extending to perhaps $500 \mathrm{pc}$ from the star. It therefore may include material in the line of sight to the pulsar.

Guélin: In order to compute the radius of Strömgren spheres around the $\mathrm{O}$ and $\mathrm{B}$ type stars, I have assumed that the density is equal to $1 \mathrm{~cm}^{-3}$ inside the $\mathrm{O}$ star $\mathrm{H}_{\mathrm{Il}}$ regions and $0.5 \mathrm{~cm}^{-3}$ inside the $\mathrm{B}$ star $\mathrm{H}$ II regions. These values are small compared to those in the known $\mathrm{H}$ II regions (Murdin and Sharpless). If a few stars sit in an intercloud region, the density inside the corresponding $\mathrm{H}$ II regions may be lower than $0.5 \mathrm{~cm}^{-3}$, the $\mathrm{H}$ II regions will be larger, and some of the lines of sight assumed to be 'clean' may well cross them. However, because of this low density, the contribution to the dispersion measure will very probably be smaller, in the mean, than that of the more dense Hil regions supposed to lie on the "nonclean' lines of sight. The argument thus still holds. If all the stars sit in an intercloud medium with $n_{\mathrm{e}} \ll 0.5$. $\mathrm{cm}^{-3}$, of course this is no longer valid. 